\title{
Combined MEK and STAT3 inhibition reprograms the tumor microenvironment to overcome immunotherapy resistance in pancreatic cancer
}

Jashodeep Datta ${ }^{\# 1,2}$, Purushottam Lamichhane ${ }^{\# 1,3}$, Xizi Dai $^{\# 1}$, Siddharth Mehra1 ${ }^{1}$ Austin R. Dosch $^{1}$, Fanuel Messaggio ${ }^{1}$, Oliver Umland ${ }^{4}$, Brent A. Willobee ${ }^{1}$, Michael N. VanSaun ${ }^{1,6}$, Peter J. Hosein ${ }^{2,5}$, Nagaraj S. Nagathihalli ${ }^{1,2}$, Nipun B. Merchant ${ }^{1,2}$

${ }^{1}$ Department of Surgery, University of Miami Miller School of Medicine, Miami, FL, USA.

2 Sylvester Comprehensive Cancer Center, University of Miami Miller School of Medicine, Miami, FL, USA

${ }^{3}$ LECOM School of Dental Medicine, Bradenton, FL, USA.

${ }^{4}$ Diabetes Research Institute, University of Miami Miller School of Medicine, Miami, FL, USA.

${ }^{5}$ Department of Medicine, University of Miami Miller School of Medicine, Miami, FL, USA.

${ }^{6}$ Department of Cancer Biology, University of Kansas Medical Center, Kansas City, KS, USA.

\#Equal authors

Running Title: MEKi/STAT3i overcomes immunotherapy resistance in PDAC

Keywords: PD-1 blockade; RAS pathway; MEK inhibition; STAT3 inhibition; pancreatic cancer; immune checkpoint inhibition;

Financial Support: This work was supported by the NIH R01 CA161976, Pancreatic Cancer Action Network (PanCan)-AACR Translational Research Grant (15-65-25-MERC), NIH T32 CA211034 and Sylvester Comprehensive Cancer Center to N. B. Merchant; KL2 career development grant by the Miami Clinical and Translational Science Institute (CTSI) under NIH Award UL1TR002736, Stanley Glaser Foundation, American College of Surgeons Franklin Martin Career Development Award, and Association for Academic Surgery Joel J. Roslyn Faculty Award to J. Datta; NIH NCI R21 CA209536, and American Cancer Society IRG 98-27713 to N.S. Nagathihalli, Society of University Surgeons Resident Research Award (AWD005237) to A. Dosch. Research reported in this publication was supported by the $\mathrm{NCI} / \mathrm{NIH}$ Award P30CA240139.

\section{Corresponding Author:}

Nipun B. Merchant, Division of Surgical Oncology, Dewitt Daughtry Department of Surgery, Sylvester Comprehensive Cancer Center, University of Miami Miller School of Medicine, 1120 NW 14 ${ }^{\text {th }}$ Street $\mid$ CRB 410, Miami, Florida 33136

Phone: 305-243-4902

Fax: 305-243-4907

Email: nmerchant@med.miami.edu

Conflict of Interest: The authors declare no potential conflicts of interest. 


\section{ABSTRACT}

Pancreatic ductal adenocarcinoma (PDAC) is characterized by immune exclusion, stromal desmoplasia, and resistance to immune checkpoint inhibition (ICI). We have previously demonstrated that reciprocally activated RAS/RAF/MEK/ERK and JAK/STAT3 pathways mediate therapeutic resistance, while combined MEK and STAT3 inhibition (MEKi/STAT3i) overcomes this resistance in PDAC. Herein, we show that combined MEKi/STAT3i alters stromal architecture and remodels the tumor-infiltrating innate and adaptive immune compartments by downregulating immunosuppressive myeloid populations and promoting T-cell

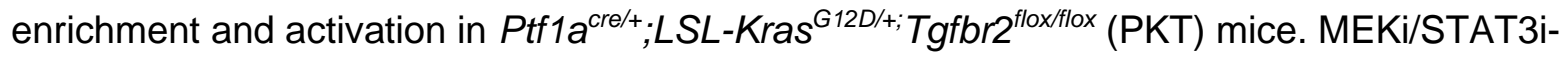
mediated reprogramming of the PDAC tumor microenvironment primes tumor-infiltrating CD8 ${ }^{+}$ T-cells for PD-1 blockade to augment their activation and functional cytotoxicity. As such, the addition of MEKi/STAT3i to PD-1 blockade dramatically inhibits tumor growth and prolongs survival, overcoming resistance to ICI, in PKT mice. Importantly, treatment of a patient with chemotherapy-refractory metastatic PDAC with MEKi (Trametinib), STAT3i (Ruxolitinib), and PD-1 inhibitor (Nivolumab) was not only well-tolerated but also yielded significant clinical benefit.

Significance: These data uncover a novel paradigm in which combined MEKi/STAT3i reprograms the stromal and immune microenvironment to overcome immunotherapy resistance in PDAC. The clinical efficacy of combined MEKi, STAT3i, and anti-PD1 treatment in chemotherapy-refractory PDAC provides encouraging signals for its translatability, and is being currently pursued in a clinical trial. 


\section{INTRODUCTION}

Pancreatic ductal adenocarcinoma (PDAC) remains a major therapeutic challenge due to its innate and acquired chemoresistance. The major contributors to therapeutic resistance that have been difficult to overcome in PDAC are mutations in the KRAS oncogene (1), the presence of a dense desmoplastic stroma that acts as a barrier to drug delivery and effector immune cell infiltration (2), and the immunosuppressive tumor microenvironment (TME) that renders the tumor resistant to immune checkpoint inhibition (ICI) immunotherapy (3).

Despite unprecedented success in other solid tumors (4), clinical trials utilizing single or dual ICI have largely been unsuccessful in PDAC patients $(5,6)$. The failure of ICI in PDAC has been attributed not only to the fibroinflammatory desmoplastic stroma in PDAC that promotes Tcell exclusion, but also to the establishment of an immunosuppressive ecosystem in the TME. Cellular elements of this ecosystem comprise innate immune populations such as tumorassociated macrophages (TAM) and myeloid-derived suppressor cells (MDSC), which induce Tcell suppression through diverse mechanisms (7). A critical unmet need in the field of PDAC immunotherapy, therefore, is the discovery of novel therapeutic strategies not only to mitigate these resistance mechanisms but also to overcome ICI resistance in PDAC.

KRAS mutations are not only the predominant oncogenic driver in over $90 \%$ of PDAC, but also promote an inflammatory program that establishes immune privilege in the TME $(1,8)$. Since targeting KRAS has remained an elusive goal, our approach has been to target downstream effectors of Ras through MEK inhibition (MEKi). However, clinical trials of MEKi have been unsuccessful in PDAC (9), likely due to the activation of resistance pathways. We have shown that MEK inhibition (MEKi) results in reciprocal activation of STAT3 signaling, which confers therapeutic resistance and continued PDAC cell growth. Combined inhibition of JAK/STAT3 (STAT3i) and MEKi overcomes this resistance mediated by parallel feedback loop activation, remodels the tumor stroma, attenuates tumor growth in patient-derived xenograft (PDX) models, and improves survival in the aggressive PDAC genetically engineered 


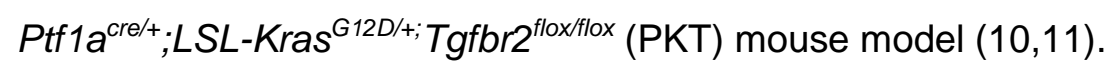

In the present study, we identify a novel paradigm in which combined MEKi/STAT3i remodels stromal fibrosis to enhance $\mathrm{CD}^{+}$and $\mathrm{CD}^{+} \mathrm{T}$-cell recruitment while attenuating immunosuppressive TAMs and MDSCs in the PDAC TME. The addition of MEKi/STAT3i to PD1 blockade overcomes $\mathrm{ICI}$ resistance by significantly augmenting anti-tumor responses and dramatically improving survival in PKT mice, compared with vehicle treatment or anti-PD-1 monotherapy. The addition of MEKi/STAT3i to PD-1 blockade not only augments the recruitment of activated and memory T-cell populations, but also improves their degranulating capacity and functional cytotoxicity. Importantly, we demonstrate the clinical efficacy and tolerability of combined Trametinib (MEKi), Ruxolitinib (STAT3i), and Nivolumab (anti-PD1) treatment in a patient with chemotherapy-refractory PDAC, providing strong evidence of the translatability of this therapeutic strategy in PDAC patients.

\section{RESULTS}

\section{Combined MEKi/STAT3i remodels stromal fibrosis and promotes an anti-tumor immune microenvironment.}

We have previously shown that combined MEKi/STAT3i, decreases tumor burden and improves survival in an aggressive GEMM of PDAC (10). We extend these results here to show that combined MEKi/STAT3i significantly remodels the tumor stroma as evidenced by decreased trichrome blue, smooth muscle actin ( $\alpha-S M A)$, and Sirius Red staining of pancreatic tumor sections in PKT mice (Fig. 1A). Moreover, MEKi/STAT3i significantly reduces intratumoral mucin content (Alcian Blue staining) and cellular proliferation (Ki67), as well as increases microvessel density (CD31 staining) compared with vehicle treatment (Supplementary Fig. S1A). To assess the immune repercussions of stromal remodeling with MEKi/STAT3i compared with vehicle treatment, flow cytometric analysis of PKT tumors ( $n=7-10$ mice per arm) revealed a significant decrease in $\mathrm{CD} 11 \mathrm{~b}^{+}$myeloid cells and $\mathrm{F} 4 / 80^{+}$ 
macrophages, as well as a concomitant increase in $\mathrm{CD}^{+}$and $\mathrm{CD} 8^{+}$tumor-infiltrating $\mathrm{T}$-cell populations (Fig. 1B). These changes were confirmed with immunofluorescence (Supplementary Fig. S1B).

To comprehensively assess global immune changes associated with MEKi/STAT3i in PKT mice, we performed multiparametric immune profiling of pooled pancreatic tumor samples utilizing time-of-flight mass cytometry (CyTOF). Compared with vehicle treatment, MEKi/STAT3i, resulted in broad-based changes in the innate $\left(C D 11 b^{+}\right)$, adaptive $\left(C D 4^{+}\right.$and $\mathrm{CD}^{+}$), and humoral $\left(\mathrm{CD19}{ }^{+}\right)$immune compartments (Fig. 1C). Specifically, compared with vehicle treatment, MEKi/STAT3i treatment resulted in a dramatic decrease in CD11 $\mathrm{b}^{+}$myeloid cells, F4/80+ macrophages, M2-like macrophages $\left(\mathrm{F} 4 / 80^{+} \mathrm{CD} 206^{+}\right)$, and MDSCs $\left(\mathrm{CD} 11 \mathrm{~b}^{+} \mathrm{F} 4 / 80^{-}\right.$ Ly6G $^{+} /$Ly6C $^{+}$) (Fig. 1D), as well as a significant increase in proportions of total $\left(\mathrm{CD}^{+}\right)$, T-helper $\left(\mathrm{CD}^{+}\right)$, cytotoxic $\left(\mathrm{CD}^{+}\right)$, and activated $\left(\mathrm{CD}^{+} 9^{+}\right)$T-cell populations (Fig. 1E). MEKi/STAT3i also improved effector $\left(\mathrm{CD}_{4} 4^{+}\right)$and central $\left(\mathrm{CD} 2 \mathrm{~L}^{+}\right)$memory T-cell markers, as well as TCR $\beta$ expression, in the PDAC TME compared with vehicle treatment (Supplementary Fig. S1C). Taken together, these data suggest that combined MEKi/STAT3i reprograms the fibroinflammatory tumor stroma to promote an anti-tumor immune microenvironment by decreasing immunosuppressive myeloid cell populations and augmenting T-cell recruitment and activation.

\section{Anti-tumor effects of MEKi/STAT3i are T-cell dependent}

We have previously shown that MEKi/STAT3i results in improved survival compared with vehicle treatment or MEKi or STAT3i monotherapy in PKT mice (10). Given the aforementioned immunologic changes with MEKi/STAT3i, we sought to determine if the anti-tumor effects of this combination therapy are $\mathrm{T}$-cell dependent by performing $\mathrm{CD}^{+}$and $\mathrm{CD} 8^{+} \mathrm{T}$ cell depletion in this model concurrently with MEKi/STAT3i (Supplementary Fig. S2). Non-T-cell depleted PKT mice treated with MEKi/STAT3i showed a significant decrease in tumor weight compared with vehicle 
treatment and demonstrated $100 \%$ survival up to 80 days of treatment (Fig. 1F\&G). In contrast, T-cell depleted mice treated with MEKi/STAT3i showed no significant decrease in tumor weight compared with vehicle-treated mice (Fig. 1F), despite confirmation of pERK and pSTAT3 inhibition in tumor lysates (Fig. 1H). Furthermore, the median survival of the T-cell depleted MEKi/STAT3i mice was significantly diminished compared with MEKi/STAT3i treatment without T-cell depletion (median 60.5 vs. not reached [NR] days, respectively; log-rank p=0.0001; Fig. 1G). These results show that the decreased tumor burden and improved survival with MEKi/STAT3i in PDAC are T-cell dependent.

\section{MEKi/STAT3i-mediated alterations in the PD-1/PD-L1 axis support the addition of immune checkpoint inhibition in PDAC}

To further explore the functional status of tumor-infiltrating CD8 ${ }^{+}$T-cells in MEKi/STAT3itreated versus vehicle-treated mice, we examined CD8 ${ }^{+}$T-cell antigen experience $\left(\mathrm{PD} 1^{+}\right)$and degranulating activity $\left(\mathrm{CD} 107 \mathrm{a}^{+}\right)$in these cells. Flow cytometric (Figure 1I) and CyTOF analysis (Supplementary Fig. S1B) revealed a significant upregulation of PD1 ${ }^{+}$tumor-infiltrating $\mathrm{CD}^{+}$ T-cells in mouse tumors treated with MEKi/STAT3i compared with vehicle treatment. Despite a significant increase in antigen-experienced degranulating CD8+PD1+CD107a+ T-cells with MEKi/STAT3i treatment (Fig. 1J), we also observed an increased proportion of PD1+CD107a non-degranulating CD8 ${ }^{+}$T-cells (Fig. 1K). Moreover, we found no significant changes in PD-L1 expression in PKT tumors between vehicle treatment, MEKi or STAT3i monotherapy, and combined MEKi/STAT3i treatment (Supplementary Figure S3). Taken together, these immunologic effects associated with MEKi/STAT3i suggest that the addition of ICI to MEKi/STAT3i may overcome resistance to immunotherapy in immunologically inert PDAC.

\section{Treatment with MEKi/STAT3i overcomes resistance to PD-1 blockade}


To build on these observations, PKT mice were treated with vehicle, aPD-1 monotherapy, MEKi/STAT3i, and MEKi/STAT3i combined with aPD-1 antibody (Supplementary Fig. S4A). Consistent with our previous data (10), MEKi/STAT3i significantly reduced tumor burden compared with vehicle treatment in PKT mice. Moreover, in keeping with prior evidence in $\operatorname{Kras}^{\mathrm{G12D} /+} ; \operatorname{Trp}^{\mathrm{fl/t}} ; \mathrm{Pdx}{ }^{\mathrm{Cre}}(\mathrm{KPC})$ mice $(12)$ as well as clinical trials of ICI in PDAC patients $(5,6)$, aPD-1 monotherapy had no effect on tumor burden compared with vehicle treatment in PKT mice, further confirming that PDAC is refractory to ICI. Importantly, the addition of MEKi/STAT3i to $\alpha$ PD-1 blockade significantly reduced tumor burden compared to $\alpha$ PD-1-treated, as well as MEKi/STAT3i-treated, mice (Fig. 2A\&B). In fact, in this aggressive PKT PDAC GEMM, where the pancreata of vehicle- and $\mathrm{APD}$-1-treated mice are completely replaced by tumor by 6 weeks of age, MEKi/STAT3i treated mice show only $19 \%$ tumor area, on average, after 4 weeks of treatment ( 8 weeks of age), while the addition of MEKi/STAT3i to aPD-1 treatment maintained normal pancreas architecture with $<6 \%$ tumor area (Fig. 2A).

\section{Addition of MEKi/STAT3i to aPD-1 blockade significantly improves survival in PKT mice}

Having shown that MEKi/STAT3i drives stromal, as well as innate and adaptive immune, remodeling (Fig. 1), and that the addition of MEKi/STAT3i to aPD-1 reduced tumor burden compared with aPD-1 monotherapy, we next sought to determine if this combination therapy overcomes resistance to $\mathrm{ICI}$ to improve survival in PKT mice (treatment schema in Supplementary Fig. S4B). Confirming our previous data, MEKi/STAT3i significantly increased survival compared with vehicle treatment (median 103 vs. 44 days; $p=0.0013$ ). Consistent with the lack of response to aPD-1 monotherapy in PDAC GEMMs (13-16), we observed no difference in survival between aPD-1 monotherapy and vehicle treatment (median 45 vs. 44 days, $p=0.34$ ). Importantly, the addition of MEKi/STAT3i to PD-1 blockade dramatically improved PKT survival compared with aPD-1 monotherapy (median 181 vs. 45 days, $\mathrm{p}=0.0005$ ), as well as combined MEKi/STAT3i (median 181 vs. 103 days, $p=0.0064$; Fig. 2C). 
Furthermore, there was no difference in body weight between vehicle and treatment groups, indicating that the combination of MEKi/STAT3i with PD-1 blockade is well tolerated with no additional toxicity compared with either aPD-1 monotherapy or MEKi/STAT3i in vivo (Supplementary Fig. S4C). These results highlight the significant role of MEKi/STAT3i in overcoming resistance to $\mathrm{ICI}$ in an immunologically inert GEMM of PDAC.

\section{The addition of MEKi/STAT3i to PD-1 blockade improves recruitment, activation, and functional cytotoxicity of tumor-infiltrating T-cells}

Next, we examined the immunologic underpinnings of the dramatic improvement in survival of PKT mice treated with combined MEKi/STAT3i and aPD-1 compared with aPD-1 monotherapy. CyTOF-based immune profiling demonstrated that, compared with both vehicle treatment and aPD-1 monotherapy, the addition of MEKi/STAT3i to PD-1 blockade resulted in a significant increase in tumor-infiltrating $\mathrm{CD}^{+}$and $\mathrm{CD}^{+}{ }^{+} \mathrm{T}$-cells, as well as $\mathrm{CD}^{+} \mathrm{T}$-cells demonstrating T-cell activation markers CD44 and CD69 and central memory marker CD62L (Fig. 2D). These broad-based changes in adaptive immune populations were then corroborated by flow cytometry in the $\mathrm{CD} 4^{+}$and $\mathrm{CD} 8^{+} \mathrm{T}$-cell compartments separately. In both $\mathrm{CD} 4^{+}$and CD8 ${ }^{+}$T-cell compartments alike, compared to aPD-1 monotherapy or vehicle treatment, the addition of MEKi/STAT3i to PD-1 blockade significantly augmented proportions of activated $\left(\mathrm{CD} 69^{+}\right)$, effector memory $\left(\mathrm{CD} 44^{+} \mathrm{CD} 62 \mathrm{~L}^{-}\right)$, central memory $\left(\mathrm{CD} 44^{+} \mathrm{CD} 62 \mathrm{~L}^{+}\right)$, tissue resident memory $\left(\mathrm{CD}_{4} 4^{+} \mathrm{CD} 62 \mathrm{~L}^{-} \mathrm{CD} 103^{+}\right)$, and degranulating effector $\left(\mathrm{CD} 44^{+} \mathrm{CD} 62 \mathrm{~L}^{-} \mathrm{CD} 107 \mathrm{a}^{+}\right)$tumorinfiltrating T-cells (Fig. 2E \& Supplementary Fig. S5).

Given significant improvements in the recruitment and activation profile of tumorinfiltrating T-cells, we next investigated if the addition of MEKi/STAT3i to PD-1 blockade enhances the functional cytotoxicity of $\mathrm{CD}^{+} \mathrm{T}$-cells compared with $\mathrm{aPD}-1$ monotherapy and vehicle treatment. Using ex vivo $\mathrm{CD}^{+} \mathrm{T}$-cell:tumor cell IFN-y release assays, PKT-derived $\mathrm{EpCAM}^{+}$tumor cells were co-cultured with splenocyte-derived CD8 ${ }^{+}$T-cells from PKT mice 
treated with vehicle, aPD-1 alone and MEKi/STAT3i/ aPD-1, and the co-culture conditioned media subjected to IFN-y ELISA. The addition of MEKi/STAT3i to PD-1 blockade significantly increased the cytolytic activity of $\mathrm{CD} 8^{+} \mathrm{T}$-cells compared with aPD-1 monotherapy or vehicle treatment (Fig. 2F). Further validating these functional T-cell data, the addition of MEKi/STAT3i to PD-1 blockade significantly increased tumor-specific granzyme B levels in PKT tumor lysates, compared with aPD-1 monotherapy or vehicle treatment (Fig. 2G). These data collectively show that the addition of MEKi/STAT3i to PD-1 blockade invigorates the adaptive immune compartment to overcome T-cell dysfunction and ICI resistance in the PDAC TME. Moreover, MEKi/STAT3i, which improves tumor-infiltrating CD8+ T-cell antigen experience but does not completely rescue their degranulating capacity (Fig. 1I-K), primes an activated CD8+ T-cell $^{-}$ compartment for $\mathrm{ICl}$ to further augment the cytolytic function of PDAC-infiltrating CD8+ T-cells.

\section{Attenuation of suppressive innate immune microenvironment in PKT mice is driven by MEKi/STAT3i but not anti-PD-1 monotherapy}

Given recent evidence that myeloid cell-specific PD-1 ablation-independent of T-celldirected anti-PD-1 effects-may contribute to effector T-cell infiltration and antitumor immunity (17), we sought to investigate the differential effects of PD-1 blockade alone and combined MEKi/STAT3i/aPD-1 on the innate immune microenvironment in PKT tumors. CyTOF-based immune profiling revealed that aPD-1 monotherapy had no impact on innate immune infiltration in PKT tumors compared with vehicle treatment. The addition of MEKi/STAT3i to PD-1 blockade, however, significantly reduced the recruitment of tumor-infiltrating $\mathrm{F} 4 / 80^{+}$ macrophages, CD206+ M2-like macrophages, and Ly6GC+ MDSCs compared with aPD-1 monotherapy or vehicle treatment (Fig. 3A). Tissue-based immunofluorescence (Fig. 3B) and flow cytometric analysis (Supplementary Fig. S6) confirmed a significant decrease in CD11 $\mathrm{b}^{+}$ myeloid, F4/80+ macrophages, and Ly6G+ MDSCs following MEKi/STAT3i/aPD-1 compared with aPD-1 monotherapy or vehicle treatment. These data reinforce that the dampening of 
immunosuppressive innate populations in the PDAC TME is driven predominantly by MEKi/STAT3i without significant contributions from aPD-1 monotherapy, and may underlie the adaptive immune invigoration observed with combination MEKi/STAT3i and PD-1 blockade.

\section{Combination MEKi/STAT3i and PD-1 blockade demonstrates efficacy in a metastatic PDAC patient with chemotherapy-refractory disease}

A 69-year-old male with a remote history of Hodgkin's lymphoma presented with a resectable microsatellite stable, low-tumor mutation burden (TMB) PDAC in the pancreatic body. He received neoadjuvant fluorouracil (5-FU), leucovorin, irinotecan, and oxaliplatin (FOLFIRINOX) for three months before undergoing curative-intent distal pancreatectomy and splenectomy. Pathology revealed a margin-negative resection, and a 2-cm focus of residual adenocarcinoma with 0/33 involved lymph nodes. He then completed three months of adjuvant FOLFIRINOX. Two months following completion of chemotherapy, he was diagnosed with a local recurrence in the pancreatic bed as well as a metastatic lesion in segment VII of the liver. He initiated second-line chemotherapy with gemcitabine/nab-paclitaxel but had progression of disease with a significant reduction in performance status (Fig. 4A).

After multidisciplinary discussion, he received off-label combination treatment with MEKi Trametinib (Mekinist/Novartis, 2mg PO QD), STAT3i Ruxolitinib (Jakafi/lncyte, 5mg PO BID), and aPD-1 Nivolumab (Opdivo/Bristol-Myers Squibb, 240mg IV Q2W) (Fig. 4A). Within one month of commencing treatment, the patient experienced a dramatic improvement in clinical and performance status. Follow-up PET/CT obtained after 3 months of treatment demonstrated significant response in the pancreatic bed mass, with nearly $50 \%$ reduction in size from $43 \mathrm{~mm}$ to $23 \mathrm{~mm}$ (Fig. 4B) and a metabolic response with reduction in SUV $\max$ from 8.4 to 6.5 posttreatment in the liver metastasis on PET/CT (Fig. 4C). The regimen was well-tolerated with no reported major adverse events while on therapy. 


\section{DISCUSSION}

The present manuscript uncovers a novel treatment strategy in PDAC that modulates the immunosuppressive tumor stroma to activate functional T-cell anti-tumor immunity to overcome ICI resistance. Resistance to $\mathrm{ICI}$ is a hallmark of PDAC $(5,6)$, defined by T-cell exclusion and tissue-level immune tolerance driven by KRAS-mediated tumor-intrinsic programs $(1,8)$ as well as interwoven suppressive cellular networks comprising innate immune populations and tumor-promoting cancer-associated fibroblasts (CAF) (7). The therapeutic strategy highlighted herein of disrupting oncogenic signaling via reciprocally activated KRAS/MEK/ERK and JAK/STAT3 pathways in PDAC tumor cells using MEKi/STAT3i (10) addresses all these major barriers to overcome $\mathrm{ICI}$ resistance in an aggressive and immunologically inert PDAC GEMM. In addition to remodeling of stromal architecture which may augment intratumoral T-cell trafficking and antigen experience, the attenuation of MDSCs and M2-like TAMs observed with MEKi/STAT3i treatment may drive the pro-immunogenic T-cell landscape that sensitizes PKT mice to ICI. It is also possible that direct effects of MEKi/STAT3i on myeloid cellular signaling and/or myelopoiesis contributes to the observed adaptive immune invigoration in the PDAC TME. While the tolerogenic role of myeloid-specific STAT3 signaling has been well established in pancreatic and other solid tumors (18), the role of myeloid-specific MEK/ERK signaling (19) and its immunologic repercussions is incompletely understood. The findings herein warrant further investigation into the differential contributions of MEK/ERK and STAT3 signaling in myelopoiesis, myeloid-specific lineage commitment, and multi-directional crosstalk with disparate immune constituents of the PDAC TME.

Emerging evidence has elucidated the signaling crosstalk between tumor cells and cancer associated fibroblasts (CAF) — the predominant constituent of the PDAC stroma -in promoting hallmarks of its aggressive biology, such as epithelial-to-mesenchymal transition (EMT) and proliferative capacity (20). In addition to our group's previous discovery of 
reciprocally activated MEK/ERK (downstream of RAS) and STAT3 signaling in tumor cells promoting therapeutic resistance $(10,11)$, other groups have reported CAF-mediated factors (such as TGF- $\beta$ ) further perpetuating tumor cell-specific MEK/ERK and STAT3 co-activation (20). The impact of abrogating MEK/STAT3-driven tumor-stromal crosstalk is exemplified in the present study, with combined MEKi/STAT3i substantially remodeling stromal composition in PKT mice. The immunologic changes associated with such stromal remodeling following MEKi/STAT3i treatment, however, are more intriguing since growing evidence underscores a complex and non-linear relationship between stromal desmoplasia and effector immune infiltration in the PDAC TME (21). The immune landscape is controlled in part by diverging signaling cues from heterogenous CAF subtypes (22). It is plausible, therefore, that contextdependent targeting of tumor-promoting inflammatory CAFs_and not indiscriminate stromal depletion (23)—will be more effective in overcoming immune exclusion and therapeutic resistance in PDAC. To this end, MEKi/STAT3i may exert its immune potentiating effects by mitigating inflammatory CAF phenotypes or abolishing CAF-intrinsic tolerogenic signaling mechanisms (e.g., STAT3 signaling (24)) that orchestrate immune suppression and establish immune privilege in the PDAC TME. Ongoing work in our laboratory is dissecting how inhibition of cooperative MEK/ERK and STAT3 signaling within PDAC CAFs reshape the immune microenvironment to sensitize PDAC to ICI.

Beyond the scientific underpinnings of this novel therapeutic strategy to overcome ICI resistance in PDAC, the impressive clinical benefit and tolerability of Trametinib, Ruxolitinib, and Nivolumab in a patient with metastatic chemo-refractory PDAC is an encouraging signal of its translatability. While such dramatic responses are not uniformly expected in all patients enrolled in our upcoming phase I trial investigating this novel combination regimen in metastatic PDAC, the immunologic consequences of MEKi/STAT3i treatment in vivo will be incorporated into a 
predictive biomarker discovery platform that may inform strategic selection of future patients for these immunotherapeutic combinations.

In summary, our data present novel insight into a combinatorial targeted therapy regimen that overcomes $\mathrm{ICI}$ resistance in PDAC by restoring elements of T-cell immunosurveillance and reprogramming the immunosuppressive TME (3), laying the foundation for its clinical application in the near future.

\section{METHODS}

\section{In vivo studies}

Tumor-bearing Ptf1a ${ }^{\mathrm{Cre} /+} \mathrm{Kras}^{\mathrm{G12D/+}}$ Tgfbr2 $^{\text {floxflox }}$ (PKT) mice (provided by Dr. Harold Moses, Vanderbilt University Medical Center, Nashville, TN) were generated as previously described (25). For endpoint analysis (i.e., flow cytometry, CyTOF, histology), PKT mice were treated with vehicle $(0.5 \% \mathrm{HPMC}+0.1 \%$ Tween 80$)$, trametinib (MEKi, Novartis, 0.067 $\mathrm{mg} / \mathrm{ml} / \mathrm{mouse}$, oral gavage three times weekly), JAK1/2 inhibitor, ruxolitinib (STAT3i, TargetMol, $0.4 \mathrm{mg} / \mathrm{ml} / \mathrm{mouse}$, oral gavage three times weekly), aPD-1 antibody (BioXCell, Clone \#BE0273, $200 \mu \mathrm{g} /$ mouse, intraperitoneal injection twice weekly), or combined MEKi/STAT3i or MEKi/STAT3i with aPD1 beginning at 4-4.5 weeks of age. Mice were sacrificed after four weeks of treatment or when moribund. For survival studies, MEKi $(0.067 \mathrm{mg} / \mathrm{ml} / \mathrm{mouse})$ and STAT3i ( $0.4 \mathrm{mg} / \mathrm{ml} / \mathrm{mouse}$ ) were administered by oral gavage 5 days/week starting at 4.5 weeks of age; aPD-1 antibody dosing remained intraperitoneal injection twice weekly. Six weeks after treatment initiation, the frequencies of MEKi/STAT3i and aPD1 antibody dosing were reduced to three times weekly and once weekly, respectively, and continued until mice were moribund (Supplementary Figure S4A\&B). T-cell depletion experiments are described in

\section{Supplementary Methods.}


All animal experiments were performed in compliance with the ethical guidelines of the Institutional Animal Care and Use Committee at the University of Miami (Protocol \#18-040).

\section{Flow cytometry}

Pancreatic tumors harvested from PKT mice were enzymatically digested using solution of $0.6 \mathrm{mg} / \mathrm{ml}$ of collagenase $\mathrm{P}$ (Roche), $0.8 \mathrm{mg} / \mathrm{ml}$ Collagenase $\mathrm{V}$ (Sigma Aldrich), $0.6 \mathrm{mg} / \mathrm{ml}$ soybean trypsin inhibitor (Sigma Aldrich), and $1800 \mathrm{U} / \mathrm{ml}$ DNase I (Thermo Scientific) in RPMI medium for $20-30$ minutes at $37^{\circ} \mathrm{C}$. Samples were then washed and resuspended in cold PEB solution followed by straining through $40 \mu \mathrm{m}$ mesh filter to obtain single cell suspension. Spleen samples were processed using RBC lysis buffer, followed passage through $100 \mu \mathrm{m}$ mesh filter. Samples were frozen at $-80^{\circ} \mathrm{C}$ until further use. Prior to flow cytometry staining, samples were thawed, washed prior to incubation with FcR blocking reagent (Miltenyi Biotec), and subsequently stained with fluorescently conjugated antibodies listed in Supplementary Table S1. Live/dead cell discrimination was performed using Live/Dead Aqua (Life Technologies) or Ghost Red Dye 780 (TONBObiosciences) as per manufacturer's protocol. Cell fixation was performed using 1\% formaldehyde solution (Thermo Fisher). Flow cytometric data acquisition was performed on CytoFLEX S (Beckman Coulter) and analyzed using FlowJo v10 software (BD Life Sciences). Gating strategies are depicted in Supplementary Figure S7.

\section{Mass cytometry (CyTOF)}

Single-cell suspensions from PKT tumors were flow sorted using FACS Aria III (BD Life Sciences) to remove non-cellular contaminants. Samples were resuspended and viability staining performed using $2.5 \mu \mathrm{M}$ cisplatin (Fluidigm) working solution following by Fc receptor blocking and surface staining for $30 \mathrm{~min}$ at room temperature using tagged antibodies as depicted in Supplementary Table S2. Fixation was then performed using $1.6 \%$ formaldehyde 
solution (Thermo Fisher). Cells were suspended in $125 \mathrm{nM}$ Cell-ID Intercalator-Ir solution

(Fluidigm) overnight at $4^{\circ} \mathrm{C}$. The following day, cells were washed and resuspended in $0.1 \mathrm{XQ}$ Four Element Calibration Beads (Fluidigm) before acquisition. Data were acquired by Helios

Mass Cytometer (Fluidigm) and analyzed using FlowJo v10 and Cytobank (Beckman Coulter).

To generate viSNE plots, individual files from each treatment group were concatenated into a single file and then a subset of equal events were selected at random through FlowJo

DownSample plugin. Total number of $\mathrm{CD} 45^{+}$cells from each group were used to create viSNE through Cytobank with 2000 iterations, perplexity of 30, and theta of 0.5 .

\section{Histologic Analysis and Western Blotting \\ See Supplementary Methods}

\section{T-cell Functional Cytotoxicity Assays}

PKT mice were treated with vehicle, aPD-1, or MEKi/STAT3i with aPD-1 for 4 weeks before sacrifice and processing of tumor samples for flow cytometry. FACS-sorted viable EpCAM ${ }^{+}$ tumor cells and total splenocytes were isolated from each mouse. Tumor cells were irradiated (4 Gy for 7 minutes) and plated in a direct co-culture system with isolated splenocytes. Condition media was collected after 72 hours of co-culture and protein content determined by BCA method. Secreted IFN-y was measured by ELISA as per manufacturer's instruction (R\&D Systems).

For measurement of intratumoral Granzyme B (GzB) levels, pancreatic tumor homogenates were prepared from PKT mice treated with vehicle, aPD-1, or MEKi/STAT3i with aPD-1 for 4 weeks. Tumor lysates were quantified and $40 \mu \mathrm{g}$ protein loaded for analysis of GzB levels by ELISA (R\&D Systems).

\section{Statistical analysis}


Descriptive statistics were calculated using Prism 9.0 (GraphPad Software Inc.). Results are shown as mean \pm SEM. Multiple comparisons were performed using ANOVA followed by Tukey's multiple comparisons test. The paired two-sided Student's t test was used for two-group comparison. Survival curves were estimated using the Kaplan-Meier method and differences between groups were assessed using the log-rank test. An $\alpha$-cutoff $\leq 0.05$ was used to define statistical significance. 


\section{REFERENCES}

1. Collins MA, Bednar F, Zhang Y, Brisset JC, Galban S, Galban CJ, et al. Oncogenic Kras is required for both the initiation and maintenance of pancreatic cancer in mice. J Clin Invest 2012;122(2):639-53 doi 10.1172/JCI59227.

2. Kerr EM, Gaude E, Turrell FK, Frezza C, Martins CP. Mutant Kras copy number defines metabolic reprogramming and therapeutic susceptibilities. Nature 2016;531(7592):110-3 doi 10.1038/nature16967.

3. Beatty GL, Eghbali S, Kim R. Deploying Immunotherapy in Pancreatic Cancer: Defining Mechanisms of Response and Resistance. Am Soc Clin Oncol Educ Book 2017;37:267-78 doi 10.14694/EDBK_175232

10.1200/EDBK_175232.

4. Tang J, Yu JX, Hubbard-Lucey VM, Neftelinov ST, Hodge JP, Lin Y. Trial watch: The clinical trial landscape for PD1/PDL1 immune checkpoint inhibitors. Nat Rev Drug Discov 2018;17(12):854-5 doi 10.1038/nrd.2018.210.

5. Marabelle A, Le DT, Ascierto PA, Di Giacomo AM, De Jesus-Acosta A, Delord JP, et al. Efficacy of Pembrolizumab in Patients With Noncolorectal High Microsatellite Instability/Mismatch Repair-Deficient Cancer: Results From the Phase II KEYNOTE-158 Study. J Clin Oncol 2020;38(1):1-10 doi 10.1200/JCO.19.02105.

6. O'Reilly EM, Oh DY, Dhani N, Renouf DJ, Lee MA, Sun W, et al. Durvalumab With or Without Tremelimumab for Patients With Metastatic Pancreatic Ductal Adenocarcinoma: A Phase 2 Randomized Clinical Trial. JAMA Oncol 2019 doi 10.1001/jamaoncol.2019.1588.

7. Balachandran VP, Beatty GL, Dougan SK. Broadening the Impact of Immunotherapy to Pancreatic Cancer: Challenges and Opportunities. Gastroenterology 2019;156(7):2056-72 doi 10.1053/j.gastro.2018.12.038. 
8. di Magliano MP, Logsdon CD. Roles for KRAS in pancreatic tumor development and progression. Gastroenterology 2013;144(6):1220-9 doi 10.1053/j.gastro.2013.01.071.

9. Infante JR, Somer BG, Park JO, Li CP, Scheulen ME, Kasubhai SM, et al. A randomised, double-blind, placebo-controlled trial of trametinib, an oral MEK inhibitor, in combination with gemcitabine for patients with untreated metastatic adenocarcinoma of the pancreas. Eur $\mathrm{J}$ Cancer 2014;50(12):2072-81 doi 10.1016/j.ejca.2014.04.024.

10. Nagathihalli NS, Castellanos J, Lamichhane P, Messaggio F, Shi C, Dai X, et al. Inverse Correlation of STAT3 and MEK Signaling Mediates Resistance to RAS Pathway Inhibition in Pancreatic Cancer. Cancer Res 2018 doi 10.1158/0008-5472.CAN-18-0634.

11. Nagathihalli NS, Castellanos JA, Shi C, Beesetty Y, Reyzer ML, Caprioli R, et al. Signal Transducer and Activator of Transcription 3, Mediated Remodeling of the Tumor Microenvironment Results in Enhanced Tumor Drug Delivery in a Mouse Model of Pancreatic Cancer. Gastroenterology 2015;149(7):1932-43 e9 doi 10.1053/j.gastro.2015.07.058.

12. Winograd R, Byrne KT, Evans RA, Odorizzi PM, Meyer AR, Bajor DL, et al. Induction of Tcell Immunity Overcomes Complete Resistance to PD-1 and CTLA-4 Blockade and Improves Survival in Pancreatic Carcinoma. Cancer Immunol Res 2015;3(4):399-411 doi 10.1158/23266066.CIR-14-0215.

13. Azad A, Yin Lim S, D'Costa Z, Jones K, Diana A, Sansom OJ, et al. PD-L1 blockade enhances response of pancreatic ductal adenocarcinoma to radiotherapy. EMBO Mol Med 2017;9(2):167-80 doi 10.15252/emmm.201606674.

14. Jiang $\mathrm{H}$, Hegde S, Knolhoff BL, Zhu Y, Herndon JM, Meyer MA, et al. Targeting focal adhesion kinase renders pancreatic cancers responsive to checkpoint immunotherapy. Nat Med 2016;22(8):851-60 doi 10.1038/nm.4123.

15. Soares KC, Rucki AA, Wu AA, Olino K, Xiao Q, Chai Y, et al. PD-1/PD-L1 blockade together with vaccine therapy facilitates effector T-cell infiltration into pancreatic tumors. $\mathrm{J}$ Immunother 2015;38(1):1-11 doi 10.1097/CJI.0000000000000062. 
16. Steele CW, Karim SA, Leach JDG, Bailey P, Upstill-Goddard R, Rishi L, et al. CXCR2 Inhibition Profoundly Suppresses Metastases and Augments Immunotherapy in Pancreatic Ductal Adenocarcinoma. Cancer Cell 2016;29(6):832-45 doi 10.1016/j.ccell.2016.04.014.

17. Strauss L, Mahmoud MAA, Weaver JD, Tijaro-Ovalle NM, Christofides A, Wang Q, et al. Targeted deletion of PD-1 in myeloid cells induces antitumor immunity. Sci Immunol 2020;5(43) doi 10.1126/sciimmunol.aay1863.

18. Kortylewski M, Kujawski M, Wang T, Wei S, Zhang S, Pilon-Thomas S, et al. Inhibiting Stat3 signaling in the hematopoietic system elicits multicomponent antitumor immunity. Nat Med 2005;11(12):1314-21 doi 10.1038/nm1325.

19. Allegrezza MJ, Rutkowski MR, Stephen TL, Svoronos N, Perales-Puchalt A, Nguyen JM, et al. Trametinib Drives T-cell-Dependent Control of KRAS-Mutated Tumors by Inhibiting Pathological Myelopoiesis. Cancer Res 2016;76(21):6253-65 doi 10.1158/0008-5472.CAN-161308.

20. Ligorio M, Sil S, Malagon-Lopez J, Nieman LT, Misale S, Di Pilato M, et al. Stromal Microenvironment Shapes the Intratumoral Architecture of Pancreatic Cancer. Cell 2019;178(1):160-75 e27 doi 10.1016/j.cell.2019.05.012.

21. Carstens JL, Correa de Sampaio P, Yang D, Barua S, Wang H, Rao A, et al. Spatial computation of intratumoral T cells correlates with survival of patients with pancreatic cancer. Nat Commun 2017;8:15095 doi 10.1038/ncomms15095.

22. Ohlund D, Handly-Santana A, Biffi G, Elyada E, Almeida AS, Ponz-Sarvise M, et al. Distinct populations of inflammatory fibroblasts and myofibroblasts in pancreatic cancer. J Exp Med 2017;214(3):579-96 doi 10.1084/jem.20162024.

23. Van Cutsem E, Tempero MA, Sigal D, Oh DY, Fazio N, Macarulla T, et al. Randomized Phase III Trial of Pegvorhyaluronidase Alfa With Nab-Paclitaxel Plus Gemcitabine for Patients With Hyaluronan-High Metastatic Pancreatic Adenocarcinoma. J Clin Oncol 2020;38(27):318594 doi 10.1200/JCO.20.00590. 
24. Biffi G, Oni TE, Spielman B, Hao Y, Elyada E, Park Y, et al. IL1-Induced JAK/STAT

Signaling Is Antagonized by TGFbeta to Shape CAF Heterogeneity in Pancreatic Ductal

Adenocarcinoma. Cancer Discov 2019;9(2):282-301 doi 10.1158/2159-8290.CD-18-0710.

25. Chytil A, Magnuson MA, Wright CV, Moses HL. Conditional inactivation of the TGF-beta type II receptor using Cre:Lox. Genesis 2002;32(2):73-5 doi 10.1002/gene.10046. 


\section{FIGURE LEGENDS}

Figure 1. Combined MEKi/STAT3i inhibits stromal fibrosis, remodels the immune microenvironment, and reduces tumor growth in a T-cell-dependent manner. (A)

Trichrome blue, $\alpha-S M A$, and Sirius Red staining in tumor sections from PKT mice treated with vehicle or MEKi+STAT3i for 4 weeks; relative areas of positive staining of respective markers from tissue sections in vehicle- and MEKi/STAT3i-treated mice are indicated in adjacent histograms. (B) Levels of total myeloid $\left(\mathrm{CD} 11 \mathrm{~b}^{+}\right)$, macrophage $\left(\mathrm{F} 4 / 80^{+}\right)$, and T-cell populations $\left(\mathrm{CD}^{+}\right.$and $\left.\mathrm{CD}^{+}\right)$were compared between vehicle and MEKi/STAT3i-treated PKT mice by flow

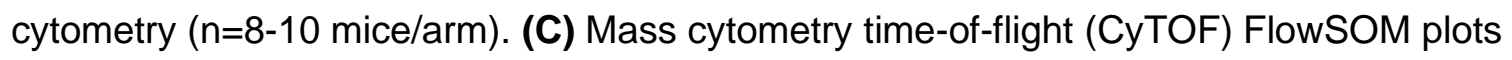
depicting changes in total myeloid $\left(\mathrm{CD} 11 \mathrm{~b}^{+}\right)$, T-cell $\left(\mathrm{CD} 4^{+}\right.$and $\left.\mathrm{CD} 8^{+}\right)$and B-cell $\left(\mathrm{CD} 19^{+}\right)$ populations in PKT mice treated with vehicle or MEKi+STAT3i ( $\mathrm{n}=7-8$ mice/arm).

Representative viSNE plots demonstrating changes in individual (D) myeloid and (E) T-cell subsets between vehicle- and MEKi+STAT3i-treated PKT mice as analyzed by CyTOF. Parent $\mathrm{CD}_{11 \mathrm{~b}^{+}}(\mathrm{D})$ and $\mathrm{CD}^{+}(\mathrm{E})$ cell populations are denoted by dashed line. PKT mice were treated with vehicle, MEKi+STAT3i, or MEKi+STAT3i following T-cell depletion with anti-CD4 and antiCD8 antibodies (scheme in Supplementary Fig. S2). Differences in (F) pancreas weight at sacrifice and (G) overall survival were compared among treatment arms. (H) Western blot demonstrating target inhibition of pERK1/2 and pSTAT3 in mice treated with MEKi+STAT3i \pm Tcell depletion. Flow cytometric analysis of PKT mice treated with vehicle or MEKi+STAT3i showing an increase in (I) PD-1+ tumor-infiltrating CD8 ${ }^{+}$T-cells, (J) CD8+PD-1+CD107a+ T-cells, and $(\mathrm{K}) \mathrm{CD}^{+}{ }^{+} \mathrm{PD}-1^{+} \mathrm{CD} 107 \mathrm{a}^{-}$T-cells in MEKi+STAT3i treated mice $(\mathrm{n}=8-10$ mice/arm). Data are shown as mean \pm SEM. Scale bar $=50 \mu \mathrm{m} .{ }^{*}, p<0.05 ;{ }^{* *}, p<0.01 ;{ }^{* *}, p<0.001$.

\section{Figure 2: Addition of MEKi/STAT3i to PD-1 blockade overcomes immune checkpoint} inhibition ( $\mathrm{ICl})$ resistance, improves survival, and augments recruitment, activation, and functional cytotoxicity of tumor-infiltrating T-cells. (A) Representative images from H\&E 
sections from PKT mice treated with vehicle, aPD-1, MEKi/STAT3i, or MEKi/STAT3i plus aPD-1 for 4 weeks (left). Both percent tumor area $(\mathbf{A}$, right) and $(\mathbf{B})$ pancreatic weight at sacrifice were compared among treatment arms ( $\mathrm{n}=5$ mice/arm). (C) Kaplan-Meier survival plot depicting overall survival in PKT mice treated with vehicle, aPD-1, MEKi/STAT3i, or MEKi/STAT3i plus aPD-1 beginning at 4-4.5 weeks of age. (D) viSNE plots demonstrating levels of tumor-

infiltrating $\mathrm{CD}^{+}$cell subsets $\left(\mathrm{CD} 4^{+}, \mathrm{CD}^{+}, \mathrm{CD}^{+} 9^{+}, \mathrm{CD}^{+} 4^{+}\right.$, and $\left.\mathrm{CD} 62 \mathrm{~L}^{+}\right)$in vehicle, $\mathrm{aPD}-1$, and combined MEKi/STAT3i/aPD-1 treated PKT mice as analyzed by CyTOF ( $n=7-8$ mice/arm). (E) Analysis by flow cytometry showing changes in total $\mathrm{CD} 3^{+}, \mathrm{CD}^{+}$, and $\mathrm{CD} 8^{+}$tumor-infiltrating $\mathrm{T}$ cells as well as levels of activated $\mathrm{CD} 9^{+}$and degranulating effector $\left(\mathrm{CD} 4^{+} \mathrm{CD} 62^{-} \mathrm{CD} 107^{+}\right)$ $\mathrm{CD}^{+} / \mathrm{CD} 8^{+}$T-cells in PKT mice treated with vehicle, aPD-1, or MEKi/STAT3i/aPD-1 ( $\mathrm{n}=7-10$ mice/arm). (F) Schematic depicting ex vivo tumor cell:splenocyte co-culture experiment. Splenocytes were isolated from PKT mice following treatment with vehicle, aPD-1, and MEKi/STAT3i/aPD-1 and co-cultured with irradiated PKT tumor cells for 72 hours. IFN-y release was determined by ELISA (right). (G) ELISA demonstrating granzyme B levels in total tumor lysate in vehicle, $\alpha \mathrm{PD} 1$, and MEKi/STAT3i/aPD-1 treatment arms ( $\mathrm{n}=3$ mice/arm). Data are shown as mean \pm SEM. Scale bar $=50 \mu \mathrm{m} .{ }^{*}, \mathrm{p}<0.05 ;{ }^{* *}, \mathrm{p}<0.01 ;{ }^{* *}, \mathrm{p}<0.001 ;{ }^{* * *}, \mathrm{p}<0.0001$.

\section{Figure 3: Differential effects on innate immune remodeling are driven by MEKi/STAT3i} with minimal contribution from anti-PD-1 monotherapy in PKT mice. (A) viSNE plots depicting CyTOF analysis of CD11 b+ myeloid cell subsets $\left(\mathrm{F} 4 / 80^{+}, \mathrm{CD}^{+} 26^{+}\right.$, and $\left.\mathrm{Ly} 6 \mathrm{G}^{+} \mathrm{C}^{+}\right)$in PKT mice treated with vehicle, $\alpha$ PD-1, and MEKi/STAT3i plus aPD-1 for 4 weeks $(n=7-8$ mice/arm). (C) Immunofluorescent staining and quantification of CD11 b+ $\mathrm{F}^{+} / 80^{+}$, and $\mathrm{Ly}_{6 \mathrm{G}}{ }^{+}$ levels in PKT mice among indicated treatment arms ( $n=3-4$ mice/arm). Data are shown as mean \pm SEM. Scale bar $=50 \mu \mathrm{m} .{ }^{*}, \mathrm{p}<0.05 ;{ }^{* *}, \mathrm{p}<0.01 ;{ }^{* * *}, \mathrm{p}<0.001 ;{ }^{* * *}, \mathrm{p}<0.0001$. 
Figure 4: Combination MEKi/STAT3i and PD-1 blockade demonstrates efficacy in a metastatic PDAC patient with chemotherapy-refractory disease. (A) Treatment timeline for patient with chemotherapy refractory PDAC prior to initiation of trametinib, ruxolitinib, and nivolumab (TRN) treatment. Pre-treatment and post-treatment PET/CT scan showing a significant reduction in size and FDG avidity of both locally recurrent tumor in pancreatic bed (B) and in a segment VII liver metastasis (C) on PET/CT imaging following 3 months of TRN treatment. 


\section{Acknowledgements}

Research reported in this publication was supported by the National Cancer Institute of the National Institutes of Health under Award Number P30CA240139. The content is solely the responsibility of the authors and does not necessarily represent the official views of the National Institutes of Health.

\section{Author Contributions:}

Conception and design: J. Datta, P. Lamichhane, X. Dai, S. Mehra, A.R. Dosch, F. Messaggio, N.S. Nagathihalli, N.B. Merchant

Development of methodology: J. Datta, P. Lamichhane, X. Dai, S. Mehra, A.R. Dosch, F. Messaggio, N.S. Nagathihalli, N.B. Merchant

Acquisition of data (provided animals, acquired and managed patients, provided facilities, etc.):

J. Datta, P. Lamichhane, X. Dai, S. Mehra, A. Dosch, F. Messaggio, B. Willobee, O. Umland, N.S. Nagathihalli, M.N. VanSaun, N.B. Merchant

Analysis and interpretation of data (e.g., statistical analysis, biostatistics, computational analysis): J. Datta, P. Lamichhane, X. Dai, S. Mehra, A. Dosch, F. Messaggio, O. Umland, B. Willobee, N.S. Nagathihalli, M.N. VanSaun, N.B. Merchant Writing, review, and/or revision of the manuscript: J. Datta, P. Lamichhane, X. Dai, A. Dosch, B. S. Mehra, F. Messaggio, Willobee, N.S. Nagathihalli, M.N. VanSaun, N.B. Merchant Administrative, technical, or material support (i.e., reporting or organizing data, constructing databases): J Datta, N.S. Nagathihalli, N.B. Merchant Study supervision: J. Datta, N.S. Nagathihalli, N.B. Merchant 
$\Delta \quad$ Vehicle MEKi+STAT3i

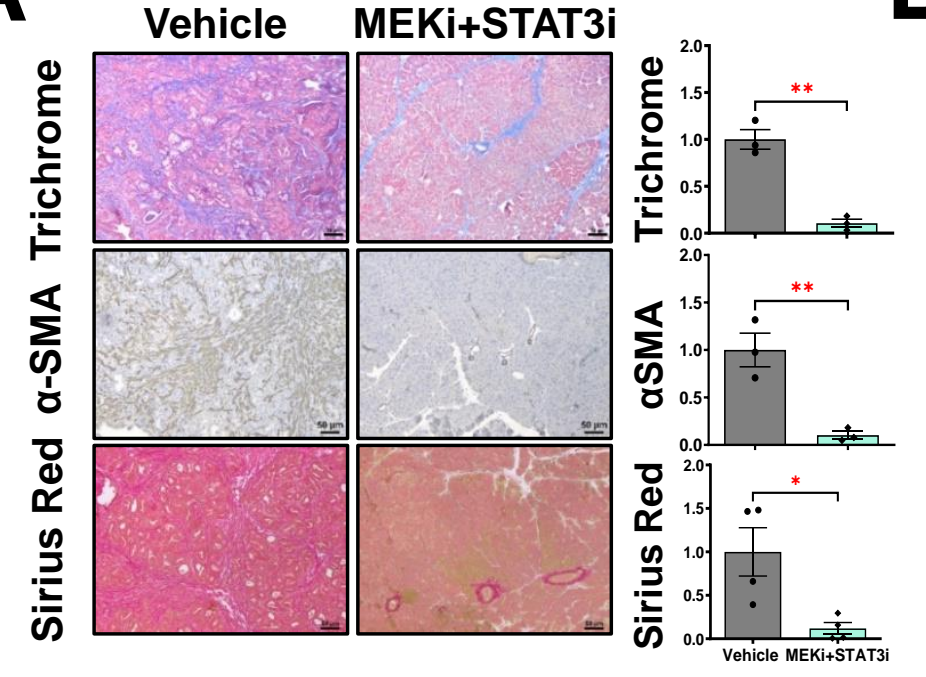

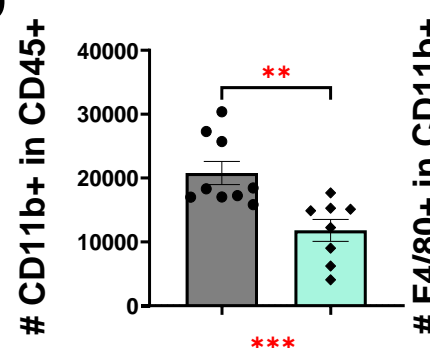

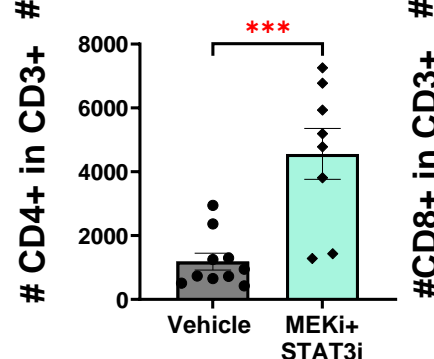

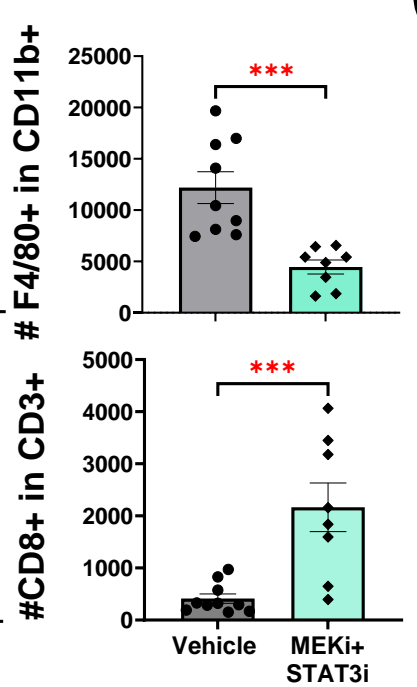

C

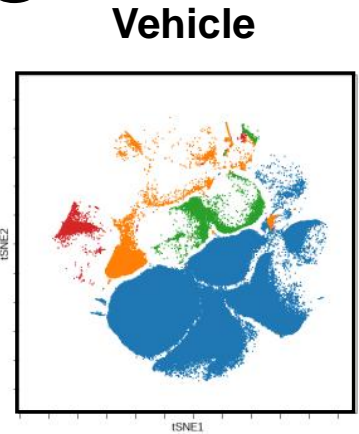

$\square$ CD11b+ Myeloid cells $\square$ CD4+ T-cells $\square$ CD19+B-cells
MEKi+STAT3i

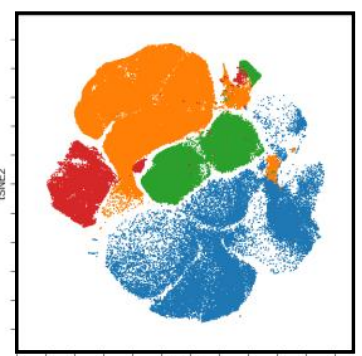

CD8+ T-cells

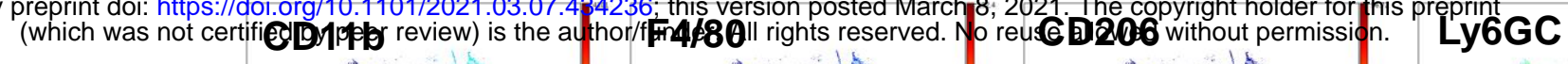

$\mathbf{E}$
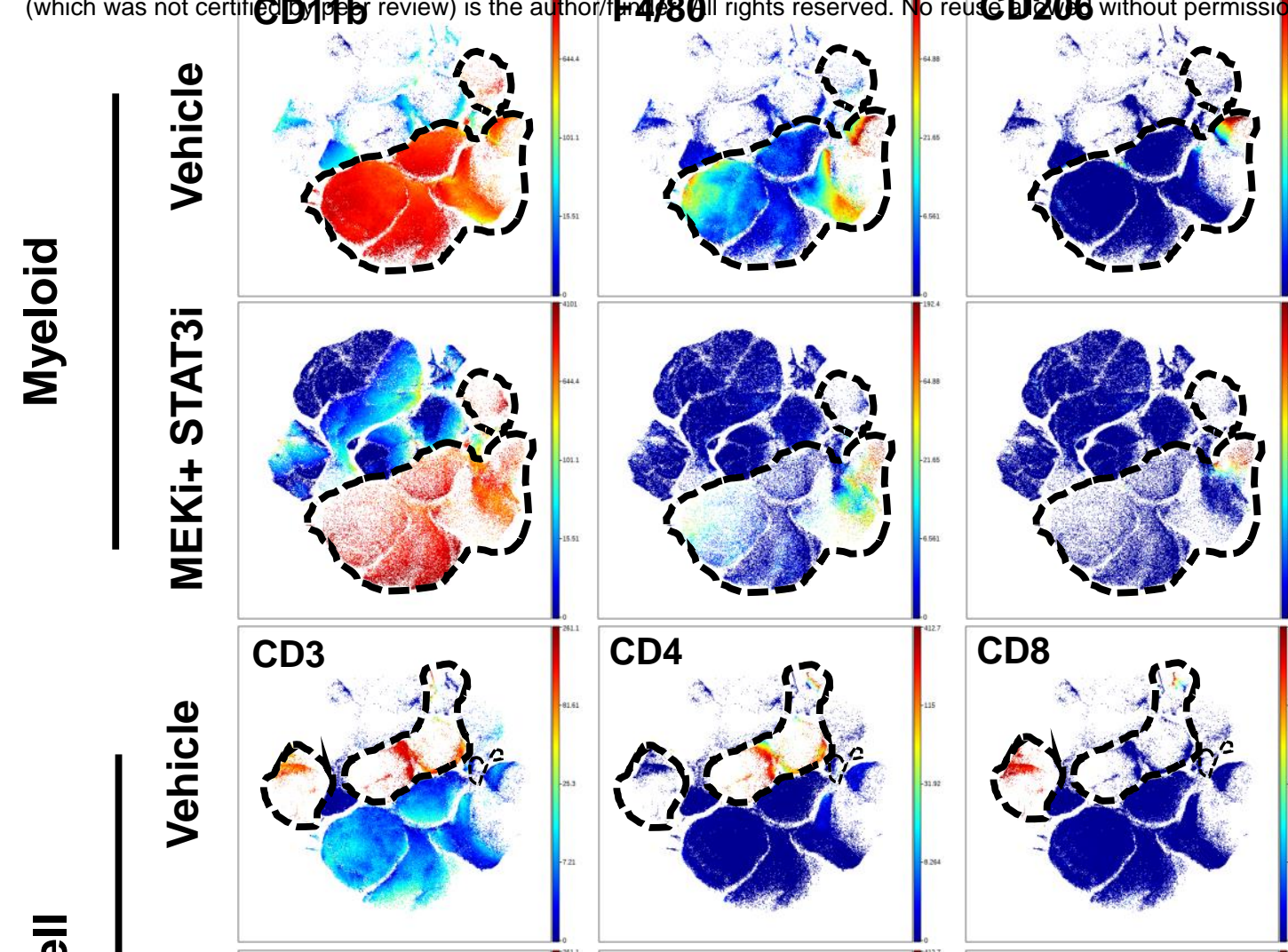

㞱
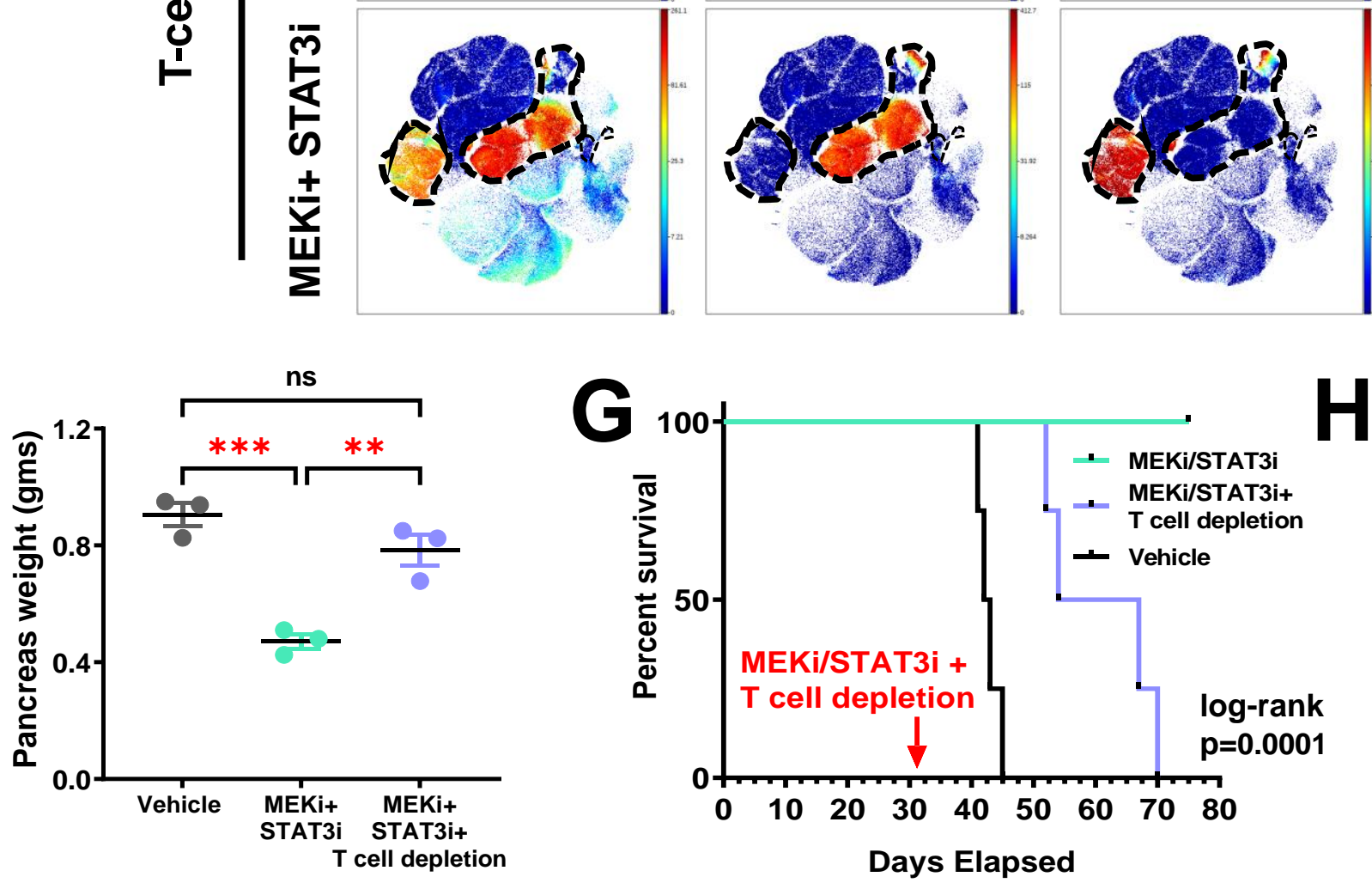

G
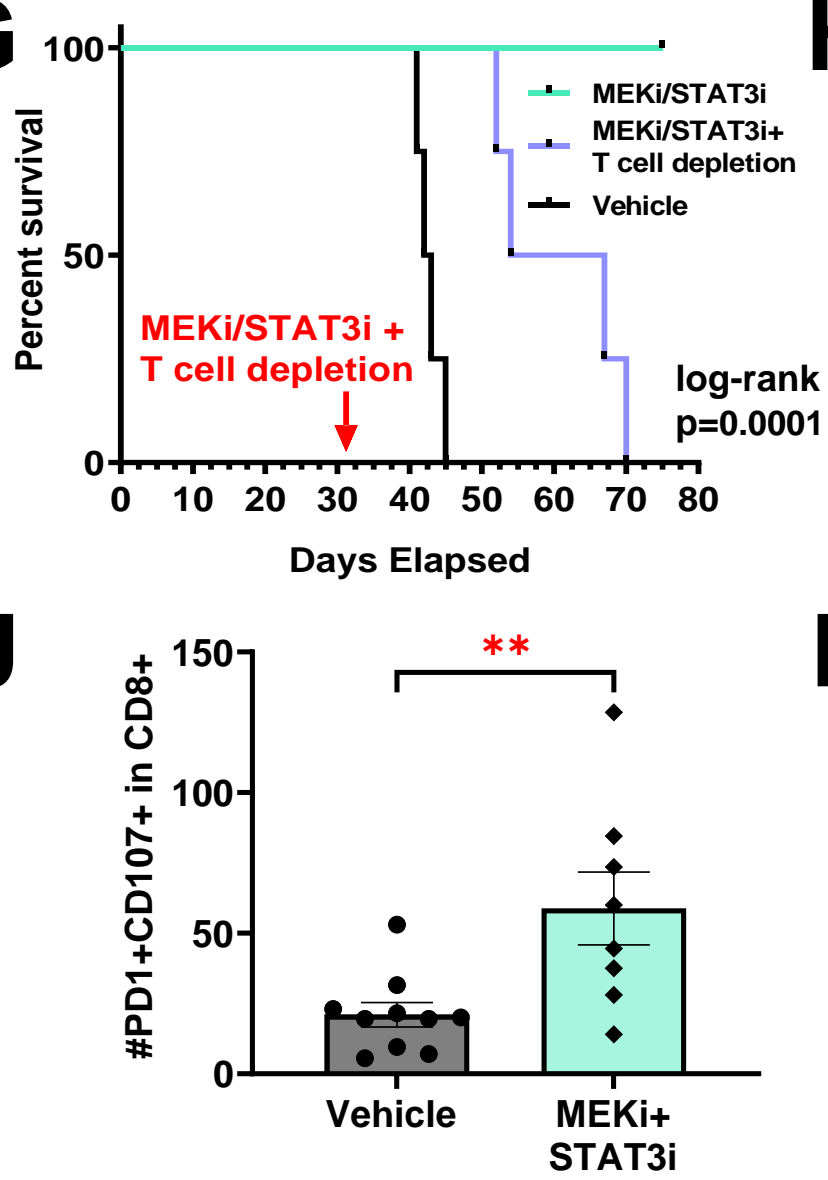

$\mathrm{H}$

$M E K i+M E K i+S T A T 3 i$

Vehicle STAT3i +T-depletion

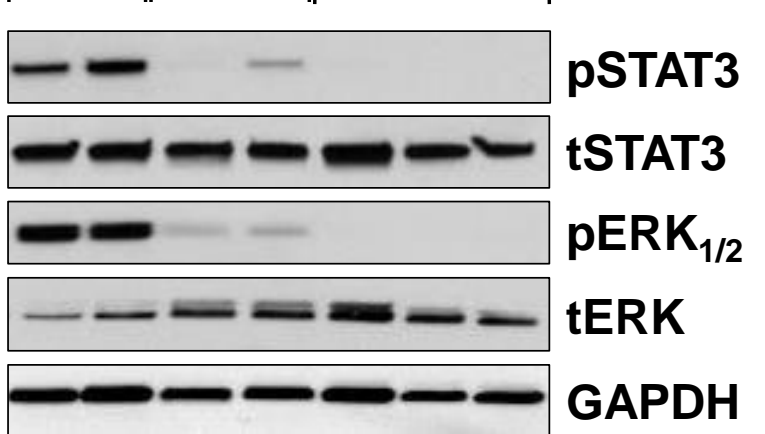

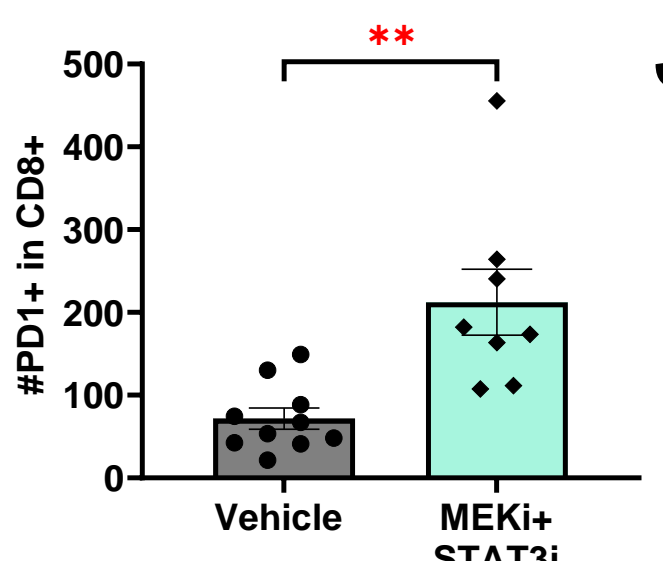

$\mathrm{K}$

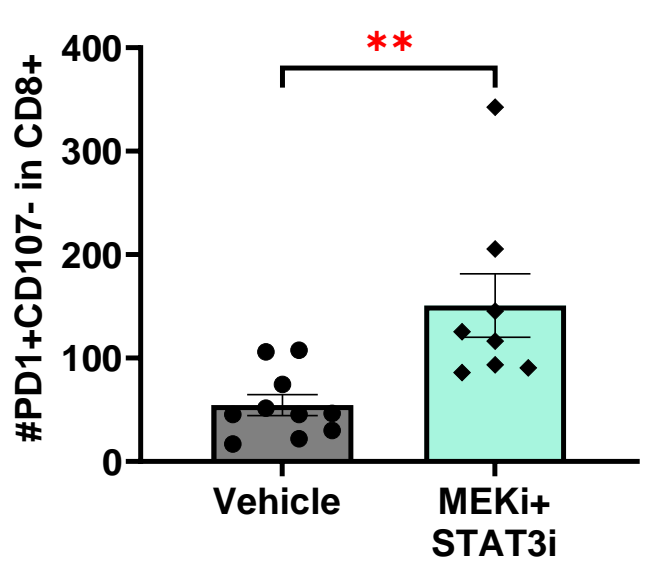



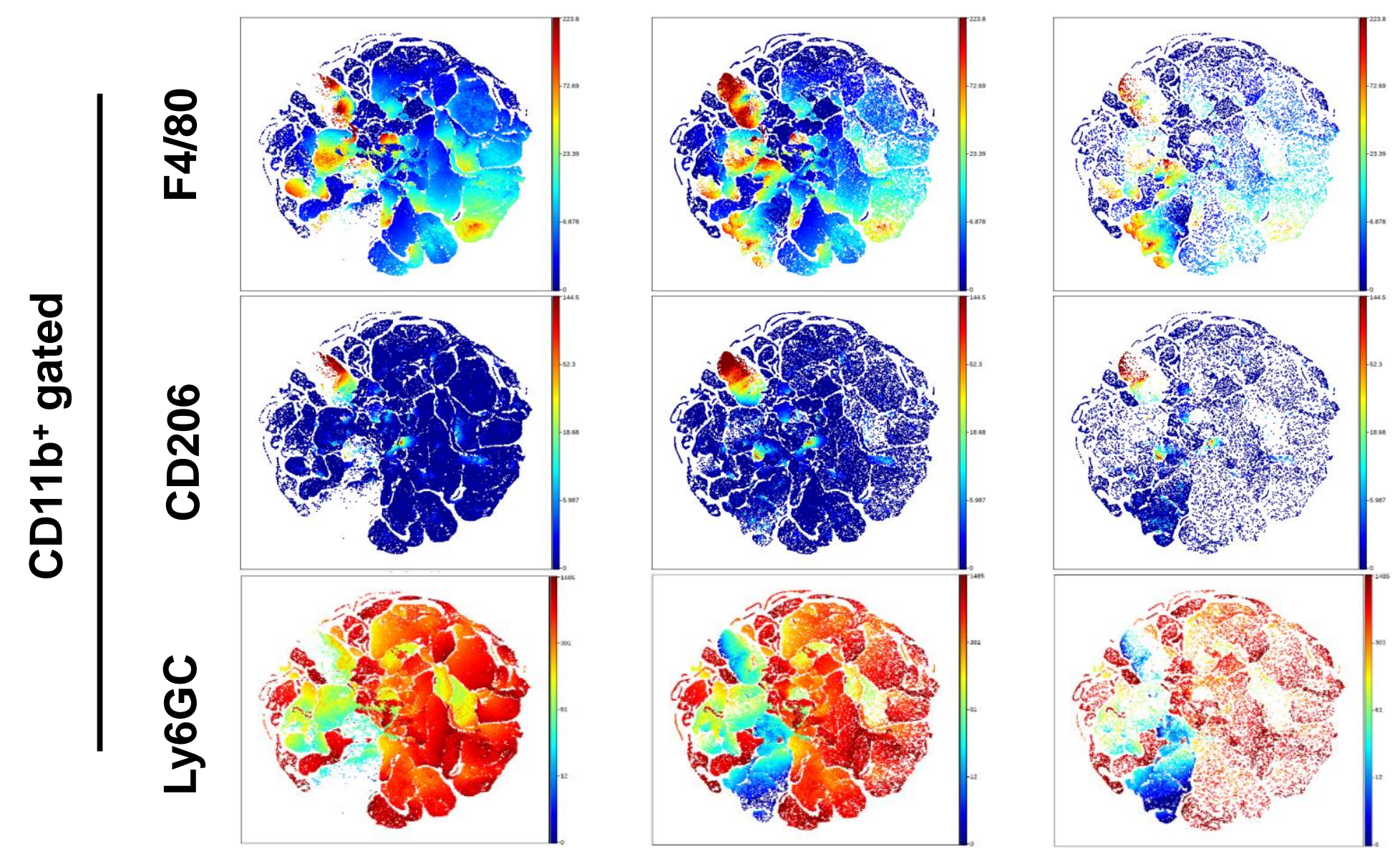

B
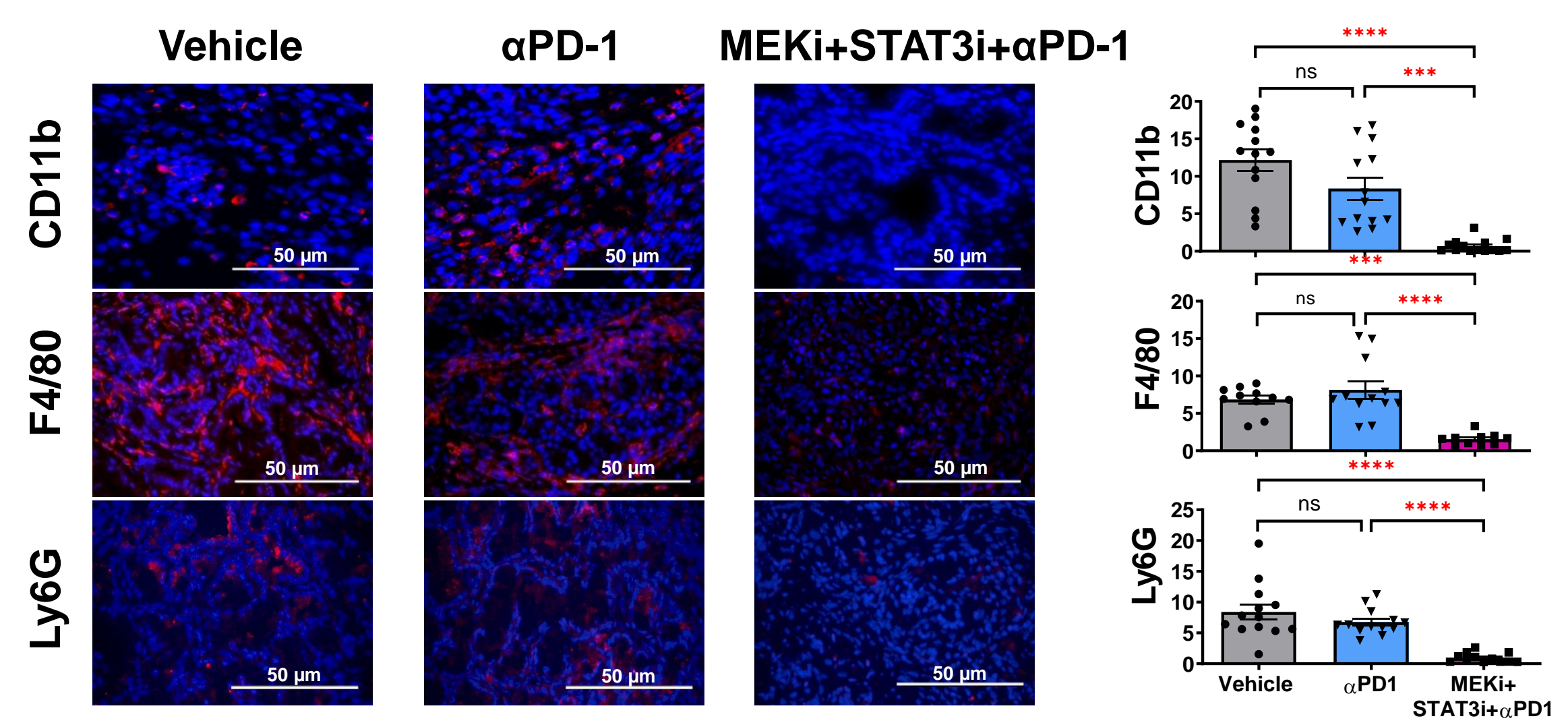
A

(months)

\section{Diagnosis}

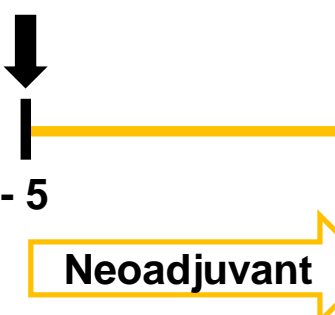

\section{Resection}

Recurrence
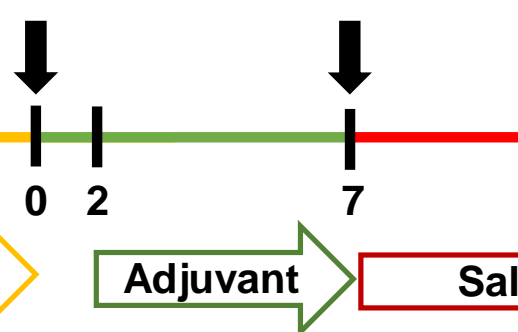

Progression

Radiologic

Response

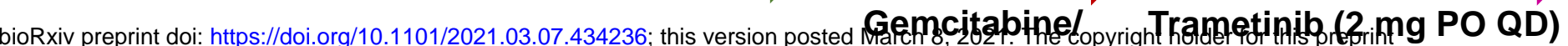

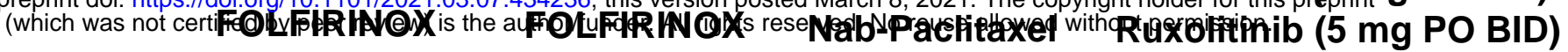
Nivolumab (240 mg IV Q2W)

B

\section{Pre-Treatment}
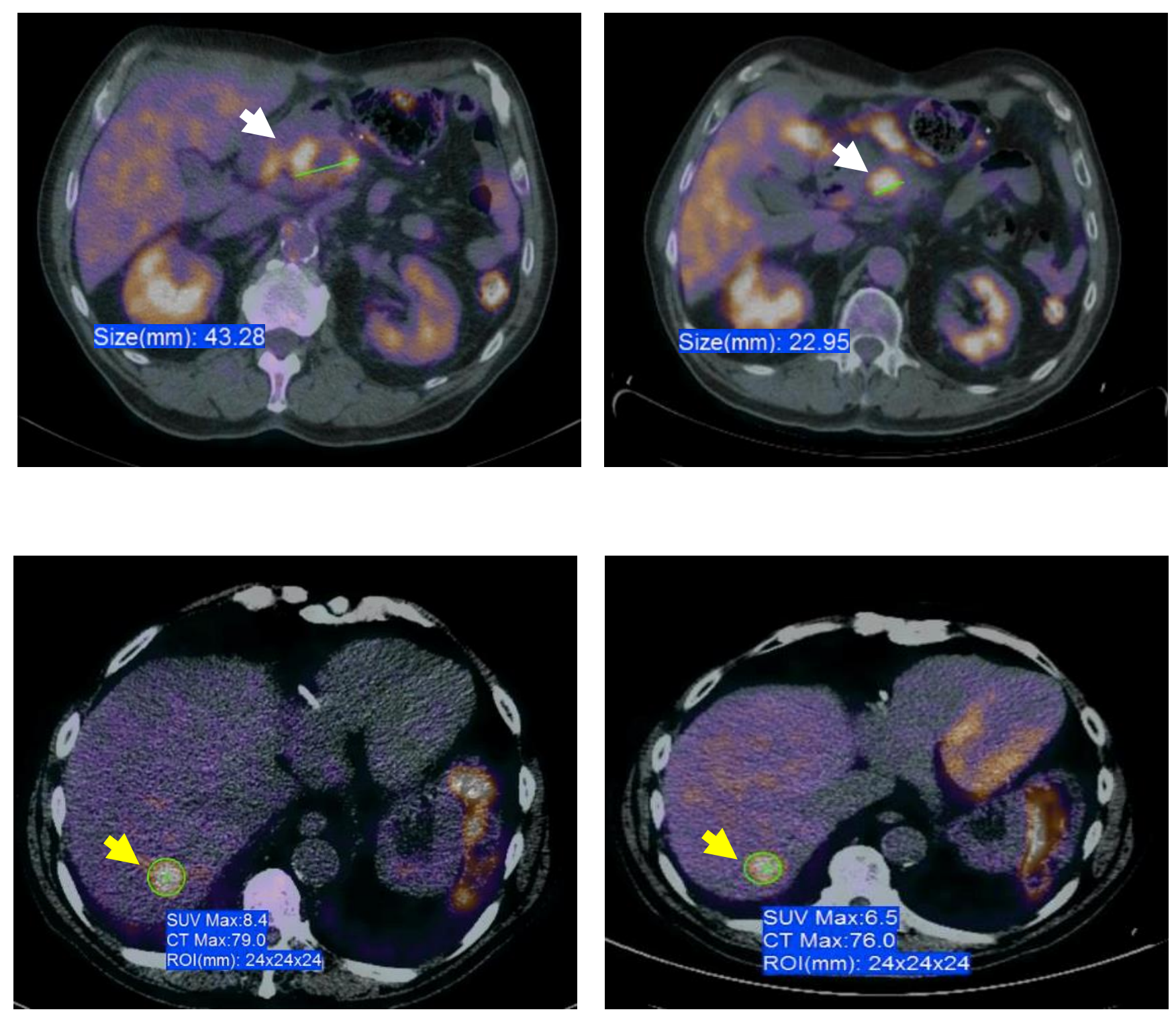

\section{Post-Treatment}

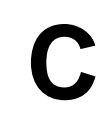

